\title{
Advances in Pediatric IBD
}

\author{
Cary M. Qualia $\cdot$ Athos Bousvaros
}

Published online: 15 May 2013

(c) Springer Science + Business Media New York 2013

\begin{abstract}
The number of children throughout the world being diagnosed with inflammatory bowel disease (IBD) is ever increasing, while recommendations regarding its diagnosis and treatment are changing rapidly. This review article aims to inform the reader of the latest advances in the field of pediatric IBD, emphasizing interesting and important new findings published over the past 12-18 months. Epidemiological findings, non-invasive testing modalities, frequently utilized treatment options, potential complications of therapy, and psychosocial concerns are all discussed herein.
\end{abstract}

Keywords Inflammatory bowel disease .

Crohn's disease · Ulcerative colitis · Pediatrics · Children · Hepatosplenic T cell lymphoma

\section{Introduction}

Inflammatory bowel disease (IBD) consists of two disorders, Crohn's disease (CD) and ulcerative colitis (UC). While CD can affect any part of the gastrointestinal tract, UC affects only the colon. It is estimated that 5 million people worldwide have IBD, with $\sim 25 \%$ of patients with $\mathrm{CD}$ and $20 \%$ of patients with UC presenting before the age of 20 years [1]. Due to the high prevalence of IBD in

C. M. Qualia $(\bowtie)$

Albany Medical Center, Albany Medical College, Pediatric

Gastroenterology, 43 New Scotland Avenue, MC-88,

Albany, NY 12208, USA

e-mail: qualiac@mail.amc.edu

A. Bousvaros

Boston Children's Hospital, Harvard Medical School,

Boston, MA, USA children, it is imperative for pediatric providers to be familiar with CD and UC, including the prevalence of these diseases and how they are currently being diagnosed and managed. Having an awareness of the potential complications associated with both diseases and their treatments is essential. Providers who care for children with IBD can assist pediatric gastroenterologists in maintaining skeletal health, warding off infections, and addressing psychosocial concerns. The purpose of this review article is to inform the reader of some of the recent developments in the care of children with IBD. The majority of the articles cited were published between July 2011 and December 2012.

\section{Recent Epidemiological Findings}

Multiple published studies have confirmed that the incidence of IBD in children is increasing. In Finland, the incidence of pediatric IBD increased from 5 per 100,000 in 1987 to 15 per 100,000 in 2003, with an average rate of increase of $6.5 \%$ per year. The incidence of CD included in this Finnish cohort increased from 2 to 5 per 100,000 over an 11 year period (1992-2003), while that of UC increased from 4 to 9 per 100,000 during the same period of time [2]. While the incidence of CD increased by $29 \%$ in northern France between 1988 and 2007, it increased by $71 \%$ in the 10-19 year old age group [3]. Children are also being diagnosed with IBD at a younger age. A Scottish study showed that the median age of diagnosis decreased from 12.7 years in the early 1990s to 11.9 years between the years of 2003 and 2008 [4]. These findings confirm a prior Canadian study by Benchimol et al. [5], which suggested that the highest increase in IBD incidence is in very young patients under age 5 . 
Children with IBD are at risk of having other immunemediated conditions, which adds to the disease burden. A study of 737 children with CD and 488 with UC showed that $\mathrm{CD}$ was associated with a higher prevalence of rheumatoid arthritis, lupus, and hypothyroidism, while UC was associated with a higher prevalence of diabetes. Children with IBD were also found to have a trend towards increased prevalence of asthma, eczema, and allergic rhinitis as compared to healthy controls [6•]. An association between IBD and celiac disease has also been described [7].

In the United States, obesity is a significant public health threat to children. Unfortunately, children with IBD living in the US are not spared from this. In a study of 1,598 such children, the prevalence of overweight/obese status was $23.6 \%$ (20.0\% for CD and $30.1 \%$ for UC and indeterminate colitis). Overweight and obese children with IBD were more likely to require surgical intervention, suggesting their disease course was more severe [8].

Lower income may also be associated with more advanced disease in children with IBD. In Canadian children younger than 18 years diagnosed with IBD between 1994 and 2004, those living in low-income neighborhoods were more likely to be hospitalized and visit the emergency department or their physician for IBD-related concerns than their counterparts living in higher-income areas. Children from low-income neighborhoods with CD were more likely to undergo intra-abdominal surgery within three years of diagnosis than those living in more affluent areas [9].

\section{Non-invasive Testing Modalities}

While endoscopic testing and mucosal biopsies remain the gold standard for diagnosing CD and UC in children, other less invasive testing modalities have emerged. Many of these are performed commonly in children suspected of having IBD or already carrying the diagnosis. These include laboratory and imaging studies, some of which are described below.

\section{Fecal Calprotectin}

Fecal calprotectin levels have been shown to be elevated in large cohorts of adult patients with IBD. A retrospective case-control study of 190 children younger than 18 years (91 with IBD and 99 without IBD) showed that fecal calprotectin level was superior to commonly utilized blood parameters such as C-reactive protein and white blood cell count $(p<0.01)$ as a screening test for IBD [10]. This test also serves as a non-invasive means of accurately determining disease severity. In a study of 35 children and adolescents with IBD (17 with UC and 18 with CD), fecal calprotectin levels were strongly correlated with endoscopic scoring systems [11]. Other newly described markers of intestinal mucosal inflammation include fecal chitinase 3-like-1 and fecal HMGB1 (high-mobility group box 1), both of which have been shown to be elevated in children with IBD [12, 13].

\section{Magnetic Resonance Enterography}

MR enterography (MRE) has proven extremely useful in the diagnosis and monitoring of patients with IBD. This testing modality may assist the clinician in differentiating acute from chronic bowel wall inflammation, inflammatory from fibrostenotic strictures, and determining whether extraintestinal disease is present. While MRE reduces radiation exposure, disadvantages of MRE include greater cost, longer examination times, and perhaps a reduction in the level of mucosal detail. Some experts have recommended MRE as the primary cross-sectional imaging modality in children being evaluated for IBD. Fast T1- and T2weighted MRI sequences and hyperosmolar biphasic oral contrast agents have allowed for high-resolution images of the bowel [14]. In a published study of 54 children with CD who underwent MRE, the most common findings of small bowel inflammation included increased contrast enhancement of the bowel wall $(93.5 \%)$, thickening of the bowel wall $(90.3 \%)$, and derangement of bowel shape with saccular formations $(25.8 \%)$ [15].

Perhaps the greatest advantage of MRE over other imaging modalities is the absence of ionizing radiation exposure. Children with IBD have been shown to have high radiation exposure over the course of their lives. In a study of 117 children with IBD ( 86 with CD and 31 with UC), the median current exposure was 15.1 millisieverts $(\mathrm{mSv})$ in $\mathrm{CD}$ and $7.2 \mathrm{mSv}$ in UC, with computed tomography and small bowel follow-through being responsible for 43 and $36 \%$ of all radiation exposures, respectively [16]. Additional information on pediatric imaging safety can be found at the patient education website www.imagegently.com.

\section{Wireless Capsule Endoscopy}

In patients suspected of having CD, wireless capsule endoscopy can assess mucosal inflammation in the midsmall bowel, potentially leading to a definitive diagnosis. Additionally, this testing modality may result in a reclassification of IBD from UC to CD. In a small study of 18 children who underwent capsule endoscopy, eight of ten suspected of having IBD were definitively diagnosed with CD after small bowel ulcerations were found. Treating physicians reported that capsule endoscopy impacted medical decision making in 13 of the 18 patients [17]. 
Wireless capsule endoscopy studies have been safely performed on children as young as two years (author's own experience). In children too young to be able to swallow the capsule, it can be endoscopically placed in the duodenum with minimal difficulty at the time of an esophagogastroduodenoscopy.

\section{Microbiome-Based Diagnostics}

Alterations exist in the oral, intestinal, and fecal microbiomes of children with IBD $[18,19]$. While microbiome alterations cannot yet be used to diagnose IBD, such evolving technology offers promise. Papa et al. [20] applied synthetic learning in microbial ecology (SLiME) analysis to fecal samples of 91 children and young adults and found that these could not only differentiate control patients from those with IBD, but could also distinguish patients with $\mathrm{CD}$ from those with UC with reasonable accuracy. In a study of 114 children, Docktor et al. [21] showed that tongue brushings from patients with $\mathrm{CD}$ showed a significant decrease in overall microbial diversity as compared with those of healthy controls using a $16 \mathrm{~S}$ rRNA-based technique known as the Human Oral Microbe Identification Microarray (significant changes were seen in Fusobacteria and Firmicutes). Additional data on sensitivity, specificity and cost will be needed to ascertain whether these tests will ever become clinically useful in the diagnosis and monitoring of IBD.

\section{Recent Data Regarding Treatment Modalities}

\section{Exclusive Enteral Nutrition}

While the vast majority of children with IBD are pharmacologically treated, it is important to remember that nutritional therapy can be very efficacious. In a published retrospective study conducted in France, 86 of 106 patients treated with either oral or continuous enteral nutritional therapy (Modulen IBD $^{\circledR}$ ) achieved remission 8 weeks into therapy. All patients showed a significant decrease in disease severity assessed by the pediatric Crohn's disease activity index as well as significant improvements in anthropometric measures and inflammatory indices. Mucosal healing was evidenced on follow-up endoscopies in a subgroup of patients. Noncompliant patients had lower mucosal healing and remission rates [22]. Gerasimidis et al. [23] showed an increase in lean but not fat mass in 17 children with active CD treated exclusively with Modulen IBD $^{\circledR}$ for 6-8 weeks. Suboptimal plasma levels of micronutrients including trace elements, vitamin C, B6, and folate found at baseline also improved during the intervention period.
Medical Therapy of IBD

\section{5-Aminosalicylates (5-ASA)}

5-ASA has been for many years a mainstay of therapy for children with UC despite there being a paucity of data to support its use. A recent prospective North American observational study of 213 patients with newly diagnosed UC who received oral 5-ASA during the first 30 days of diagnosis showed that $40 \%$ were corticosteroid-free at 1 year without requiring additional therapy [24].

\section{Budesonide}

Corticosteroids remain part of the armamentarium of gastroenterologists and are often prescribed for patients with moderate or severe IBD. Some patients fail to respond to these medications, while others are unable to wean off them without experiencing a recurrence of their symptoms. The many negative side effects associated with systemic corticosteroids may preclude their usage, and long-term treatment with these medications is best avoided. Budesonide is a corticosteroid with a high first pass metabolism through the liver, thereby reducing corticosteroid-related toxicity. Data derived from the prospective Pediatric IBD Collaborative Research Group Registry established in 2002 in North America showed that 119 of 932 (13\%) of children with newly diagnosed CD were prescribed budesonide, with 56 of the 119 (47\%) starting this medication within 30 days of diagnosis. Only 26 of the above 56 patients had disease limited to the ileum and/or ascending colon. Budesonide was used as monotherapy in $9 \%$ of the patients, in combination with 5-ASA in $77 \%$, and in combination with immunomodulators (6-mercaptopurine, azathioprine, or methotrexate) in $43 \%$. Two-thirds of the patients received budesonide for six months or less. $43 \%$ of patients required conventional corticosteroids at some point following their first course of budesonide. The authors concluded that budesonide monotherapy is rare, and that further data are required to better define the role of this medication in the treatment of pediatric CD [25].

\section{Thiopurines}

Thiopurines include 6-mercaptopurine (6-MP) and its prodrug azathioprine (AZA). These medications remain a mainstay of therapy for children with moderate to severe disease. Children with IBD appear to tolerate thiopurines better than adults despite the therapeutic response to these medications being similar in both age groups. Goodhand et al., retrospectively examined 100 pediatric and adult patients with IBD started on a thiopurine and found adverse effects causing discontinuation of these medications in 
only 1 of 50 children and 8 of 50 adults $(p<0.05)$. Corticosteroid-free remission was achieved in $30 \%$ of the children and $38 \%$ of the adults $(p=0.53)$. Interestingly, disease type did not affect remission rates [26].

One of the advantages to thiopurine usage is that metabolite levels can be measured, guiding dosage adjustment and allowing for the detection of potential toxicity. In a study of 68 patients with IBD and two with eosinophilic colitis, $68 \%$ had 6-thioguanine (6-TGN) levels outside the therapeutic range despite receiving standard thiopurine dosing. Toxicity occurred in seven patients. After metabolite measurement, management was changed in 25 of 70 patients. The ability of the leukocyte count to predict 6-TGN levels was poor [27].

Evidence suggests that low 6-TGN levels may result from noncompliance, rapid metabolism, or inadequate dose of 6-MP. Low 6-TGN correlates with poorer response. High 6-TGN levels may sometimes cause leukopenia, and high 6-methylmercaptopurine (6-MMP) levels may sometimes cause increased ALT [28]. However, given the unreliable correlation between thiopurine metabolite levels and the aforementioned laboratory features, we believe the clinician does not necessarily need to adjust the dose of 6-MP based on thiopurine metabolite levels if WBC and ALT levels are normal.

\section{Methotrexate}

Methotrexate has been shown to have modest efficacy at best in the long-term treatment of both CD and UC. In a retrospective study of 93 children with IBD, clinical remission was observed in $16 \%$ of $\mathrm{CD}$ patients and $7 \%$ of patients having UC or indeterminate colitis (IC) 24 months after initiation of therapy. Remission rates at one year were 25 and $13 \%$, respectively. The one year remission rate for $\mathrm{CD}$ patients was significantly higher in those having colonic disease. While only $14 \%$ had to discontinue treatment, $49 \%$ experienced negative side effects [29]. Many pediatric gastroenterologists are now prescribing oral methotrexate in combination with $\mathrm{TNF} \alpha$ antagonists to try to prevent antibody formation to these medications (see below). Perhaps this is the strongest indication to prescribe methotrexate for children with IBD.

\section{Tumor Necrosis Factor Alpha (TNF $\alpha)$ Antagonists}

Anti-TNF $\alpha$ agents are frequently used in the management of children with IBD. While these agents were initially recommended for the treatment of $\mathrm{CD}$ in children, the use of these medications in patients with refractory UC is now accepted. Scheduled treatment regimens have been shown to be superior to episodic treatment in pediatric CD. Using these medications in combination with other immunosuppressive medications appears to reduce immunogenicity and improve therapeutic control, but increases the risks of infection and malignancy [30].

One of the benefits to using anti-TNF $\alpha$ agents is the ability of these medications to induce disease response and/ or remission quickly in a subset of patients. Hamalainen et al., showed a significant decrease in fecal calprotectin levels two and six weeks after initiation of infliximab therapy in 36 children with IBD in Finland (median $1,150 \mu \mathrm{g} / \mathrm{g}$ at baseline to $261 \mu \mathrm{g} / \mathrm{g}$ at week $2, p<0.001$ ). This suggests that intestinal inflammation may normalize very rapidly in some children receiving infliximab (1/3 of children in this study) [31]. Short-term disease remission rates for children receiving adalimumab have also been encouraging. One study demonstrated remission rates of 24, 58 and $41 \%$ at one, six, and 12 months, respectively, following initiation of therapy in 72 patients with IBD (70 with $\mathrm{CD}$ ) treated at 19 pediatric centers [32].

A few studies have examined the long-term outcomes of children with IBD receiving these medications. In one such study conducted retrospectively, 120 patients (101 with CD and 19 with UC or IC) had received either infliximab or adalimumab. The median duration of treatment was 15 months. Eighty-nine percent of the cohort experienced short-term response associated with improvement in weight, erythrocyte sedimentation rate, C-reactive protein level, and serum albumin level. The cumulative probability of losing response to anti-TNF $\alpha$ treatment was $17 \%$ at one year, $38 \%$ at three years, and $49 \%$ at five years [33].

\section{Combination Therapy with Immunomodulators and Anti-TNF Antibodies}

As discussed above, not all patients with IBD will respond to monotherapy with either an anti-TNF $\alpha$ or immunomodulator. Additionally, though pediatric data is lacking, many adults receiving anti-TNF $\alpha$ monotherapy will develop antibodies to these medications over time, often rendering them ineffective [34•]. The decision of whether to use anti-TNF antibodies as monotherapy or in combination with an immunomodulator remains a topic of considerable controversy [35]. Considering the above, some pediatric gastroenterologists have begun to abandon monotherapy with anti-TNF $\alpha$ agents and prefer to use these medications only in combination with an immunomodulator. In a study of 14 children with CD cared for at the Mayo Clinic pediatric IBD center who had received concomitant methotrexate and anti-TNF $\alpha$ therapy after failing anti$\mathrm{TNF} \alpha$ therapy alone, seven patients $(50 \%)$ entered clinical remission within an average of six weeks after starting methotrexate. Only one patient discontinued therapy due to adverse events. Four patients became infected with Clostridium difficile, which complicated therapy [36]. 
Therefore, while combination therapy with an immunomodulator and anti-TNF $\alpha$ agent may be more efficacious than treatment with an anti-TNF $\alpha$ agent alone, the increased degree of immunosuppression associated with combination therapy may result in the acquisition of certain infections. In our experience, combination therapy with an anti-TNF $\alpha$ agent and methotrexate is safe, well tolerated, and preferable to anti-TNF $\alpha$ monotherapy. Many of our patients who had been tried on anti-TNF $\alpha$ monotherapy developed antibodies to these agents over time, which led to treatment failure and discontinuation of the anti-TNF $\alpha$ agent.

\section{Probiotics}

Although enteric bacteria have been shown to play a crucial role in the development of IBD, there is a paucity of data confirming the efficacy of probiotics in the treatment of children with these disorders. That being said, probiotics may be helpful. Oliva et al., randomized 31 children and adolescents with mild to moderate UC to receive either Lactobacillus (L) reuteri or placebo enemas daily for eight weeks. Mayo scores (consisting of clinical and endoscopic features) and histological scores significantly decreased in the L. reuteri group, but not the placebo group. Additionally, IL-10 levels significantly increased and IL- $1 \beta$, TNF- $\alpha$, and IL- 8 levels significantly decreased only in the L. reuteri group. In other words, rectal infusion of $L$. reuteri was shown to be effective in improving mucosal inflammation and changing mucosal expression of certain cytokines involved in the pathogenesis of IBD [37].

\section{Surgical Management of IBD}

Surgical intervention is often necessary for children with IBD. Indications for surgery include disease unresponsive to medical therapy, complications of the disease that cannot be managed medically (e.g., abscesses in patients with $\mathrm{CD}$ that fail to respond to antibiotics), and other specific situations (e.g., patients with $\mathrm{CD}$ having single terminal ileal or short-segment colonic strictures).

Barrena et al., described the operations, complications, and outcomes of a relatively large group of children with IBD who had been treated in Madrid, Spain between 1992 and 2009. Eighty-five of the 192 patients had CD, 107 had UC, and three had IC. Twelve of the 85 CD patients ( $15 \%$ ) required 13 resections, one stricturoplasty, one transplanation and six other operations including three permanent enterostomies for anorectal involvement. Twenty-nine of the 107 UC patients (26\%) required 87 operations including nine emergent colectomies for toxic megacolon or severe hemorrhage and 28 restorative proctocolectomies and ileoanostomies. Complications occurred in $40 \%$ of the above surgeries [38]. One of the major complications seen in children suspected of having UC who undergo colectomy is the development of complications of $\mathrm{CD}$ following surgery. Unfortunately, despite the best efforts of physicians, the possibility of patients with severe inflammatory colitis having CD rather than UC cannot always be ruled our preoperatively. Mortellaro et al., reported nine of 68 patients having a change in their diagnosis from $\mathrm{UC}$ to $\mathrm{CD}$ following total colectomy and ileal pouch anal anastamosis by either a two- or three-stage approach. Seven of these patients required subsequent surgeries due to complications of $\mathrm{CD}$, including two needing anastomotic dilations, 4 requiring fistulotomies, and one requiring drainage of a perianal abscess [39].

It is important to note that many of the surgeries performed on children with IBD are now done via minimally invasive approaches. Mattioli et al., reported on 16 patients who underwent a total of 25 procedures. Twelve of the patients had UC, three had CD, and one had IC. Eleven underwent staged laparoscopic subtotal colectomy (LSTC) followed by J-pouch ileorectal anastamosis (JPIRA), while three underwent combined LSTC and JPIRA. All procedures included a protective ileostomy. None needed to be converted to open. Complications were observed in six ( $24 \%)$ of patients. The majority of these involved adhesions that were treated laparoscopically [40].

In children with UC or IC failing medical therapy and undergoing colectomy, preoperative immunosuppression does not appear to be associated with increased postoperative complications. In a study of 45 children with UC and six with IC, $88 \%$ had received corticosteroids, $51 \%$ had received thiopurines, and $4 \%$ had received calcineurin inhibitors in the 30 days prior to colectomy. Within 90 days of surgery, $65 \%$ had been exposed to infliximab. There was no increased incidence of early or late complications in the patients receiving thiopurines or calcineurin inhibitors within 30 days or infliximab within 90 days as compared to those who had not received these medications within the same period of time [41].

\section{Lymphoma Risk in Children with IBD}

In children with IBD, thiopurine use has been associated with a slightly increased risk of lymphoma. Ashworth et al., identified two male patients who developed lymphoma out of 1,374 children with IBD. One of these patients had Hodgkin lymphoma and the other had anaplastic large cell lymphoma. Both were receiving thiopurines but had not yet received a biologic agent at the time they were diagnosed with lymphoma. The incidence rate of lymphoma for patients having received thiopurines was determined to be 4.5 per 10,000 patient-years, as compared 
to the expected rate of 0.58 per 10,000 patient-years. This equates to a standardized incidence ration of 7.51 [42•].

Hepatosplenic T cell lymphoma (HSTCL) is of specific concern in the pediatric population as it primarily affects males younger than 35 years and is uniformly fatal. In a review of 36 patients reported, 20 had received infliximab and a thiopurine and 16 had received thiopurine monotherapy. None of the patients with HSTCL had received only anti-TNF $\alpha$ therapy. The authors concluded that thiopurines should only be given to young male patients when a clear benefit is expected, such as in early stage disease in treatment naïve patients [43•]. That being said, lymphoma is a rare complication of thiopurine usage, and the efficacy of thiopurines in the treatment of IBD has been well described [44]. We recommend using these medications with caution in young males, and warning families of the increased risk of lymphoma associated with thiopurines.

\section{Skeletal Health}

Clinicians can attempt to preserve skeletal health in their pediatric patients with IBD by ensuring adequate calcium and Vitamin D intake and avoiding prolonged corticosteroid usage.

A study of 80 patients with IBD (51 with UC, 26 with CD, three with IC) who had undergone DEXA scans compared bone mineral density to that of age matched controls. The authors found that the children with IBD had lower lumbar spine and whole body bone mineral density. A cumulative prednisolone dosage of $>150 \mathrm{mg} / \mathrm{kg}$ for the preceding three years increased the risk for low whole body bone mineral density. Vertebral fractures were found in $11 \%$ of patients but only $3 \%$ of controls $(p=0.02)$ [45]. We recommend ordering DEXA scans for children with IBD who have received prolonged or repeated courses of corticosteroids, those at an increased risk of fracture for some other reason (eg., low calcium or $25 \mathrm{OH}$ vitamin $\mathrm{D}$ level), and those who have sustained multiple fractures.

Kappelman et al., attempted to determine whether fracture risk in children with IBD is affected by geographical location. Of the 733 children with CD and 488 with UC included in the study, there were no significant differences in fracture rate between those residing in the Northeast or Midwest regions of the US versus the South [46].

\section{Immunizations}

Primarily due to the immunosuppressive medications they frequently receive, children with IBD are thought to be at in increased risk of contracting different infections, some of which are preventable through vaccination. Multiple studies have shown that a relatively large percentage of children with IBD are not appropriately immunized to certain vaccine preventable infections and that some of these individuals are not able to mount an anamnestic response.

Hepatitis B virus (HBV) reactivation can occur in patients receiving infliximab. Moses et al., prospectively evaluated 100 children with IBD on infliximab and found that while 87 were vaccinated against HBV, only 49 of these $87(56 \%)$ had immunity to HBV as defined by an anti-hepatitis B surface antibody level of $\geq 10 \mathrm{mIU} / \mathrm{ml}$. Thirty-four patients received booster immunizations, but only $76 \%$ of these showed an anamnestic response. The authors recommended screening children for HBV immunity at the time of IBD diagnosis since a subset of these patients are not immune to $\mathrm{HBV}$ at the time of starting immunosuppressive therapy [47]. In two other recently published studies, lack of sufficient immunity to other vaccine-preventable illnesses including varicella and influenza B was found in a relatively large percentage of pediatric patients $[48,49]$. In the deBruyn study referenced above, children with IBD were less likely to mount an immunogenic response to the influenza $B$ vaccination than controls (53 vs. $81 \%$ ), and were also less likely to achieve serologic protection (79 vs. $100 \%$ ) [49].

\section{Psychosocial and Behavioral Concerns}

Children with IBD have been shown to be at risk of having multiple psychological disorders. A study of 36 adolescents ages 12-17 revealed clinical levels of anxiety in $22 \%$ and depressive symptoms in $30 \%$ [50]. Children with IBD may also have poorer school functioning, more absences from school [51], and a lower health-related quality of life as compared to their peers without IBD [52]. Clinicians caring for children with IBD should be aware of the above and should request the assistance of mental health professionals when necessary.

\section{Conclusion}

The prevalence of IBD in children is increasing. As such, it is essential for pediatric providers to be familiar with how these children are diagnosed and treated. Non-invasive testing modalities such as fecal calprotectin, MRE, and wireless capsule endoscopy are frequently utilized in the diagnostic workups of children and adolescents suspected of having IBD. Children with IBD receiving corticosteroids, immunomodulators, and/or biologic agents are at increased risk of developing certain complications 
including thin bones, infections, and even malignancies. Surgery is a viable and often life-saving option for some children with IBD and minimally invasive approaches have reduced the morbidity and mortality associated with these procedures. By working closely with pediatric gastroenterologists and staying up to date with the pediatric IBD literature, primary care physicians can improve the physical and emotional well being of children with IBD.

Disclosure Cary M. Qualia received payment for the development of educational presentations from Warner Chilcott. Athos Bousvaros reports no conflicts of interest.

\section{References}

Papers of particular interest, published recently, have been highlighted as:

- Of importance

1. Abdullah BA, Gupta SK, Croffie JM, et al. The role of esophagogastroduodenoscopy in the initial evaluation of childhood inflammatory bowel disease: a 7-year study. J Pediatr Gastroenterol Nutr. 2002;35(5):636.

2. Lehtinen $\mathrm{P}$, Ashorn $\mathrm{M}$, Iitanen $\mathrm{S}$, et al. Incidence trends of pediatric inflammatory bowel disease in Finland, 1987-2003, a nationwide study. Inflamm Bowel Dis. 2011;17(8):1778-83.

3. Chouraki V, Savoye G, Dauchet L, et al. The changing pattern of Crohn's disease incidence in norther France: a continuing increase in the 10- to 19-year old age bracket (1988-2007). Aliment Pharmacol Ther. 2011;33(10):1133-42.

4. Henderson P, Hansen R, Cameron FL, et al. Rising incidence of pediatric inflammatory bowel disease in Scotland. Inflamm Bowel Dis. 2012;18(6):999-1005.

5. Benchimol EI, Guttman A, Griffiths AM, et al. Increasing incidence of pediatric inflammatory bowel disease in Ontario, Canada: evidence from health administrative data. Gut. 2009;58(11): 1490-7.

6. - Kappelman MD, Galonko JA, Porter CQ, et al. Association of paediatric inflammatory bowel disease with other immune-mediated diseases. Arch Dis Child. 2011;96 Suppl 11:1042-6. This was a large database study that showed a clear association between IBD and other autoimmune disorders.

7. Virta LJ, Kholo KL. The risk of contracting pediatric inflammatory bowel disease in children with celiac disease, epilepsy, juvenile arthritis and type 1 diabetes-a nationwide study. J Crohn's colitis. 2013;7(1):53-7.

8. Long MD, Crandall WV, Leibowitz IH, et al. Prevalence and epidemiology of overweight and obesity in children with inflammatory bowel disease. Inflamm Bowel Dis. 2011;17(10): 2162-8.

9. Benchimol EI, To T, Griffiths AM, et al. Outcomes of pediatric inflammatory bowel disease: socioeconomic status disparity in a universal-access healthcare system. J Pediatr. 2011;158(6):960-7. e1-4.

10. Henderson P, Casey A, Lawrence SJ, et al. The diagnostic accuracy of fecal calprotectin during the investigation of suspected pediatric inflammatory bowel disease. Am J Gastroenterol. 2012;107(6):941-9.

11. Aomatsu T, Yoden A, Matsumoto K, et al. Fecal calprotectin is a useful marker for disease activity in pediatric patients with inflammatory bowel disease. Dig Dis Sci. 2011;56(8):2372-7.
12. Aomatsu $\mathrm{T}$, Imaeda $\mathrm{H}$, Matsumoto $\mathrm{K}$, et al. Faecal chitinase 3-like-1: a novel biomarker of disease activity in paediatric inflammatory bowel disease. Aliment Pharmacol Ther. 2011; 34(8):941-8.

13. Vitali R, Stronati L, Negroni A, et al. Fecal HMGB1 is a novel marker of intestinal mucosal inflammation in pediatric inflammatory bowel disease. Am J Gastroenterol. 2011;106(11): 2029-40.

14. Chalian M, Ozturk A, Oliva-Hemker M, et al. MR enterography findings of inflammatory bowel disease in pediatric patients. AJR. 2011;196:W810-6.

15. Torkzad MR, Ullberg U, Nystrom N, et al. Manifestations of small bowel disease in pediatric Crohn's disease on magnetic resonance enterography. Inflamm Bowel Dis. 2012;18(3):520-8.

16. Sauer CG, Kugathasan S, Martin DR, et al. Medical radiation exposure in children with inflammatory bowel disease estimates high cumulative doses. Inflamm Bowel Dis. 2011;17(11): 2326-32.

17. Gralnek IM, Cohen SA, Ephrath H, et al. Small bowel capsule endoscopy impacts diagnosis and management of pediatric inflammatory bowel disease: a prospective study. Dig Dis Sci. 2012;57(2):465-71.

18. Hansen R, Russell RK, Reiff C, et al. Microbiota of de-novo pediatric IBD: increased Faecalibacterium prausnitzii and reduced bacterial diversity in Crohn's but not in ulcerative colitis. Am J Gastroenterol. 2012;107(12):1913-22.

19. Kellermayer R, Mir SA, Nagy-Szakal D, et al. Microbiota separation and $\mathrm{C}$-reactive protein elevation in treatment-naïve pediatric granulomatous Crohn disease. J Pediatr Gastroenterol Nutr. 2012;55(3):243-50.

20. Papa E, Docktor M, Smillie C, et al. Non-invasive mapping of the gastrointestinal microbiota identifies children with inflammatory bowel disease. PLoS One. 2012;7(6):e39242.

21. Docktor MJ, Paster BJ, Abramowicz S, et al. Alterations in diversity of the oral microbiome in pediatric inflammatory bowel disease. Inflamm Bowel Dis. 2012;18(5):935-42.

22. Rubio A, Pigneur B, Garnier-Lengline H, et al. The efficacy of exclusive nutritional therapy in paediatric Crohn's disease, comparing fractionated oral vs. continuous enteral feeding. Aliment Pharmacol Ther. 2011;33(12):1332-9.

23. Gerasimidis K, Talwar D, Duncan A, et al. Impact of exclusive enteral nutrition on body composition and circulating micronutrients in plasma and erythrocytes of children with active Crohn's disease. Inflamm Bowel Dis. 2012;18(9):1672-81.

24. Zeisler B, Lerer T, Markowitz J, et al. Outcome following aminosalicylate therapy in children newly diagnosed with ulcerative colitis: a prospective multi-center registry experience. J Pediatr Gastroenterol Nutr. 2012;. doi:10.1097/MPG.0b013e31826ac41a.

25. Otley A, Leleiko N, Langton C, et al. Budesonide use in pediatric Crohn disease. J Pediatr Gastroenterol Nutr. 2012;55(2):200-4.

26. Goodhand JR, Tshuma N, Rao A, et al. Do children with IBD really respond better than adults to thiopurines? J Pediatr Gastroenterol Nutr. 2011;52(6):702-7.

27. Armstrong L, Sharif JA, Galloway P, et al. Evaluating the use of metabolite measurement in children receiving treatment with a thiopurine. Aliment Pharmacol. 2011;34(9):1106-14.

28. Benkov K, Lu Y, Patel A, et al. Role of thiopurine metabolite testing and thiopurine methyltransferase determination in pediatric IBD. J Pediatr Gastroenterol Nutr. 2013;56(3):333-40.

29. Willot S, Noble A, Deslandres C. Methotrexate in the treatment of inflammatory bowel disease; an 8-year retrospective study in a Canadian pediatric IBD center. Inflamm Bowel Dis. 2011; 17(12):2521-6.

30. Yang LS, Alex G, Catto-Smith AG. The use of biologic agents in pediatric inflammatory bowel disease. Curr Opin Pediatr. 2012;. doi:10.1097/MOP.0b013e3283574154. 
31. Hamalainen A, Sipponen T, Kolho KL. Infliximab in pediatric inflammatory bowel disease rapidly decreases fecal calprotectin levels. World J Gastroenterol. 2011;17(47):5166-71.

32. Russell RK, Wilson ML, Loganathan S, et al. British Society of Paediatric Gastroenterology, Hepatology and Nutrition survey of the effectiveness and safety of adalimumab in children with inflammatory bowel disease. Aliment Pharmacol Ther. 2011;33(8):946-53.

33. Assa A, Hartman C, Weiss B, et al. Long-term outcome of tumor necrosis factor alpha antagonist's treatment in pediatric Crohn's disease. J Crohns Colitis. 2012;. doi:10.1016/j.crohns.2012. 03.006.

34. -Oussalah A, Cheveaux JB, Fay R, et al. Predictors of infliximab failure after azathioprine withdrawal in Crohn's disease treated with combination therapy. Am J Gastroenterol. 2010;105(5): 1142-9. This study emphasized the risk of loss of response to biologics when immunomodulators are withdrawn.

35. Bern EM, Bousvaros A. Loss of response to biologics versus increased risk of lymphoma in children with inflammatory bowel disease: the clinician's conundrum. Expert Rev Clin Immunol. 2013;9(2):117-27.

36. Absah I, Faubion WA Jr. Concomitant therapy with methotrexate and anti-TNF-alpha in pediatric patients with refractory Crohn's colitis: a case series. Inflamm Bowel Dis. 2012;18(8):1488-92.

37. Oliva S, DiNardo G, Ferrari F, et al. Randomised clinical trial: the effectiveness of Lactobacillus reuteri ATCC55730 rectal enema in children with active distal ulcerative colitis. Aliment Pharmacol Ther. 2012;35(3):327-34.

38. Barrena S, Martinez L, Hernandez F, et al. Surgical treatment of chronic inflammatory bowel disease in children. Pediatr Surg Int. 2011;27(4):385-90.

39. Mortellaro VE, Green J, Islam S, et al. Occurrence of Crohn's disease in children after total colectomy for ulcerative colitis. J Surg Res. 2011;170(1):38-40.

40. Mattioli G, Pino-Prato A, Barabino A, et al. Laparoscopic approach for children with inflammatory bowel diseases. Pediatr Surg Int. 2011;27(8):839-46.

41. Schaufler C, Lerer T, Campbell B, et al. Pre-operative immunosuppression is not associated with increased post-operative complications following colectomy in children with colitis. J Pediatr Gastroenterol Nutr. 2012;55(4):421-4.

42. - Ashworth LA, Billett A, Mitchell P, et al. Lymphoma risk in children and young adults with inflammatory bowel disease: analysis of a large single-center cohort. Inflamm Bowel Dis. 2012;18 Suppl 5:838-43. This study highlighted the rarity of lymphoma in a large pediatric cohort and associated this with exposure to medication.

43. - Katlyar DS, Osterman MT, Diamond RH, et al. A systematic review of factors that contribute to hepatosplenic T-cell lymphoma in patients with inflammatory bowel disease. Clin Gastroenterol Hepatol. 2011;9 Suppl 1:36-41. This study reviewed all documented cases of HSTCL at the time and found an association with thiopurine therapy in young males.

44. Markowitz J, Grancher K, Kohn N, et al. A multicenter trial of 6-mercaptopurine and prednisone in children with newly diagnosed Crohn's disease. Gastroenterology. 2000;119(4):895-902.

45. Laakso $\mathrm{S}$, Valta $\mathrm{H}$, Verhasalo $\mathrm{M}$, et al. Impaired bone health in inflammatory bowel disease: a case-control study in 80 pediatric patients. Calcif Tissue Int. 2012;91(2):121-30.

46. Kappelman MD, Galanko JA, Porter CQ, et al. Risk of diagnosed fractures in children with inflammatory bowel diseases. Inflamm Bowel Dis. 2011;17(5):1125-30.

47. Moses J, Alkhouri N, Shannon A, et al. Hepatitis B immunity and response to booster vaccination in children with inflammatory bowel disease treated with infliximab. Am J Gastroenterol. 2012;107(1):133-8.

48. Ansari F, Baker RD, Patel R, et al. Varicella immunity in inflammatory bowel disease. J Pediatr Gastroenterol Nutr. 2011; 53(4):386-8.

49. de Bruyn JC, Hilsden R, Fonseca K, et al. Immunogenicity and safety of influenza vaccination in children with inflammatory bowel disease. Inflamm Bowel Dis. 2012;18(1):25-33.

50. Reigada LC, Bruzzese JM, Benkov KJ, et al. Illness-specific anxiety: implications for functioning and utilization of medical services in adolescents with inflammatory bowel disease. J Spec Pediatr Nurs. 2011;16(3):207-15.

51. Mackner LM, Bichmeier RM, Crandall WV. Academic achievement, attendance, and school-related quality of life in pediatric inflammatory bowel disease. J Dev Behav Pediatr. 2012;33(2):106-11.

52. Ross SC, Strachan J, Russell RK, et al. Psychosocial functioning and health-related quality of life in paediatric inflammatory bowel disease. J Pediatr Gastroenterol Nutr. 2011;53(5):480-8. 